\title{
ÉNERGIE ET TEMPÉRATURE $\left({ }^{(}\right)$;
}

Par II. Max PLANGK.

Invité à présenter, en ce lieu illustré par une série d'excellentes découvertes scientifiques, une des questions qui ont fait l'objet de mes propres travaux, je crois répondre de mon mieux à l'honneur qui m'est fait, en exposant devant vous un problème qui se trouve précisément au centre des recherches thermodynamiques, le problème de la relation entre la température et l'énergie. J'ai choisi ce sujet d'autant plus volontiers qu'il me fournit l'occasion d'apporter mon tribut de louanges, dans la ville même où il a exercé son action, à la mémoire de l'immortel Henri-Victor Regnault, à qui la thermodynamique doit la détermination de ses données numériques les plus importantes, et dont le monde des physiciens achève à peine de fêter le centenaire.

Quand on regarde de loin, la question des rapports entre la température et l'énergie semble des plus simples et des plus élémentaires; et elle paraît aussi facile à résoudre qu'à poser. J'espère pouvoir néanmoins montrer que réellement il n'en est pas ainsi. Fn réalité, ce problème recèle de grandes difficultés et des énigmes mystérieuses. Enfin tout récemment, un point de vue nouveau s'est fait jour, qui nous permettra d'approcher un peu plus près de la solution définitive de ce problème fondamental de la thermodynamique.

On n'a jamais trop répété que les recherches exactes en thermodynamique datent de l'époque à laquelle on a su distinguer les concepts de température et de quantité de chaleur. Le thermometre et le calorimetre sont devenus les instruments de mesure les plus importants de la thermodynamique. Tous deux ont reçu avec le temps des perfectionnements remarquables, et avec la précision des mesures est allée de pair la précision des définitions, sans laquelle la mesure la plus délicate n'aurait aucune valeur. En effet, tandis que la chaleur était présentée comme une forme particulière de l'énergie, et que la connaissance de l'équivalent mécanique de la chaleur permettait de l'exprimer en quantités absolues d'énergie,

(1) Conférence faite à la Société française de Plıysiıjue, le 21 arril 1911.

J. de Phys., วّ série, t. I. (Mai 1911.) 
une définition de la température parfaitement exacte et propre aux mesurages de précision, était déduite de l'application à la chaleur moléculaire, d'une part, à la chaleur rayonnante d'autre part, du second principe de la thermodynamique, le principe de CarnotClausius. Nous pouvons donc admettre à bon droit que les quantités de chaleur, aussi bien que les températures, peuvent être déterminées avec une précision, limitee seulement par la qualité des instruments, dont le perfectionnement se poursuit d'ailleurs sans cesse.

Mais s'il semble possible de résoudre cette première question avec toute l'approximation désirable, il nous reste encore à nous demander quelle est la relation générale qui existe entre la température et l'énergie. - En se plaçảnt simplement au point de vue de l'énergétique, on pourrait dire peut-être : "La température est un facteur de l'énergie. La température est à l'énergie calorifique ce que la force est au travail mécanique et ce que le potentiel est à l'énergie électrique. La différence de température de deux corps indique le sens des échanges de chaleur entre ces deux corps, exactement de la même façon que la force mécanique donne la direction du mouvement, ou la différence de potentiel le sens du courant électrique. "

Mais, en parlant de la sorte, on néglige un point essentiel. Le mouvement peut se faire aussi bien dans le sens opposé à la force, un courant électrique peut circuler dans le sens opposé à la chute de potentiel, tandis qu'un flux d'énergie calorifique se produisant en sens contraire de la chute de température est absolument impossible. L'existence d'une grandeur douée des propriétés que possède la température, est déjà un fait unique dans toute la physique. Le fait, que deux.corps, en équilibre calorifique avec un troisième, sont encore en équilibre calorifique entre eux, n’est pas un lait qui va de soi-même, c'est une circonstance très remarquable et très importante. En effet, il ne se rencontre rien danalogue dans le cas des équilibres électriques. On le reconnaît de suite lorsque, prenant une baguette de cuivre et une autre de zinc, on les plonge par un bout dans de l'acide sulfurique étendu, et qu'on réunit alors les deux autres bouts par un conducteur métallique. On n'obtient pas un état d'équilibre, mais un courant électrique, qui dure jusqu'à ce que la surface du cuivre se soit notablement modifiée.

On sait que toutes les relations particulières exprimant les équi- 
libres thermodynamiques peuvent être établies, d'une façon complète et absolument conforme avec l'expérience, en partant du second principe de la thermodynamique. Mais, dans le problème qui nous occupe, nous n'avons pas affaire seulement avec ce principe. La thermodynamique pure reste digne de tous nos suffrages, - elle a enrichi la science physique de découvertes inestimables, - mais il ne nous est plus possible et nous n'avons plus le droit de nous en tenir à ce qu'elle nous apprend. Car elle nous renseigne aussi peu sur les grandeurs et les relations entre les grandeurs des constantes caractéristiques des propriétés thermodynamiques des corps, que sur le temps nécessaire à l'évolution des phénomènes irréversibles, tels que la conduction et le rayonnement calorifique, la diffusion et les réactions chimiques. D'après la thermodynamique pure, le rapport des deux chaleurs spécifiques pourrait être 2 aussi bien que 100 , et la conductibilité calorifique d'un gaz pourrait être indifféremment 100.000 fois plus grande ou 100.000 fois plus petite que celle d'un métal.

Dans l'occurrence, les considérations atomistiques sont les seules qui nous puissent mettre en avant; et, au premier rang, vient la théorie cinétique des gaz. Cette théorie nous enseigne, comme conséquence de la loi de Gay-Lussac et Avogadro, que la température d'un gaz parlait est représentée par l'énergie moyenne du mouvement de chaque molécule, quel que soit le poids moléculaire.

Cette loi, simple et facilement accessible à l'intuition, semble déjà apporter un peu de lumière dans la grosse question que nous avons posée de la relation entre l'énergie et la température. Elle est en effet évidemment susceptible d'être généralisée pour les cas des gaz non parfaits, des vapeurs, des liquides et des solides. 11 suffit d'admettre seulement qu'il y a équilibre calorifique entre deux corps quand les molécules particulières des deux corps, placés l'un contre l'autre, possèdent la même énergie moyenne de mouvement. Il est facile de s'en rendre compte, en se représentant que les molécules des deux corps se choquent mutuellement et échangent ainsi leurs énergies, de telle sorte que finalement il s'établit un certain équilibre statistique, dans lequel les énergies moyennes des mouvements se sont égalisées de part et d'autre.

En fait, L. Boltzmann et J.-Ir. Gilbs, s'aidant de considérations relatives aux probabilités, sont arrivés à déduire des équations générales de Hamilton une loi générale, qu'on appelle aujourd'hui 
principe de l'équipartition de l'énergie. Il résulte de cette loi que, dans un équilibre statistique quelconque relatif à un système dépendant d'un grand nombre de variables, à chaque variable indépendante qui influe sur l'énergie du système doit être attribuée la même quantité d'énergie.

Or parmi les variables indépendantes caractéristiques de l'état d'un corps solide, liquide ou gazeux, il faut toujours compter, d'après la théorie cinétique, les composantes de la vitesse de ses molécules. Par suite, dans un système constitué par un nombre quelconque de corps solides, liquides ou gazeux et se trouvant en état d'équilibre statistique, l'énergie moyenne correspondant à chacue composante de la vitesse de chaque molécule est la même, d'après la loi de l'équipartition de l'énergie, pour tout l'ensemble du système. Il est donc évident que la condition thermodynamique de l'équilibre calorifique se trouve en parfait accord avec la loi de la mécanique statistique, si la température d'un corps est prise, d'une façon tout à fait générale, comme la mesure de l'énergie cinétique meyenne d'une composante quelconque de la vitesse del'une quelconque de ses molécules, ou, pour parler d'une façon plus générale encore, comme la mesure de l'énergie moyenne, correspondant à l'une quelconque des variables indépendantes caractéristiques du corps.

De cette sorte il semble bien que la loi de l'équipartition de l'énergie contienne la réponse définitive à la question de la relation entre l'énergie et la température ; et l'on doit avouer que l'extrème simplicité de cette loi, d'ailleurs toute irtuitive, devait facilement amener les physiciens à lui attribuer un rôle fondamental dans la thermodynamique. Et cette manière de voir s'autorise encore de diverses vérifications frappantes qu'elle a rencontrées.

Si la température exprime en même temps l'énergie moyenne, correspondant à une variable particulière, on obtiendra l'énergie totale d'un corps, en multipliant sa température par le nombre des variables indépendantes qui déterminent son état. La capacité calorifique à volume constant est alors donnée immédiatement par le nombre de ses variables indépendantes, ou autrement dit par le nombre de degrés de liberté du corps en question. Circonstance encore plus particulière, avec les unités ordinairement employées pour la quantité de la chaleur, la température et la molécule-gramme, la chaleur moléculaire a encore une valeur numérique égale au nombre de degrés de liberté de la molécule réelle. 
Dans la cas d'un gaz monoatomique par exemple, si lon considère les atomes comme des points matériels, et si l'on fait abstraction de leurs attractions mutuelles, les seuls degrés de liberté, qui interviennent dans l'expression de l'énergie, sont les trois composantes de la vitesse de l'atome. Par suite, avec les unités usuelles, la chaleur atomique d'un tel gaz considéré sous un volume constant est égale à 3. Dans le cas d'un corps solide, au contraire, il faut ajouter, aux trois composantes de la vitesse d'un atome, les trois coordonnées qui mesurent le déplacement de l'atome par rapport à la position d'équilibre et déterminent ainsi son énergie potentielle. Par suite la chaleur atomique d'un corps solide est égale à 6 , ce qui s'accorde d'assez près avec la loi de Dulong et Pelit. Les écarts par rapport à cette loi, et en particulier l'accroissement de la chaleur spécifique avec la température, observé pour toutes les substances, s'expliqueraient par l'introduction de nouveaux degrés deliberté, dus à un plus grand relâchement des liaisons de l'atome dans le groupement moléculaire. Le fait que cet accroissement ne se produit pas brusquement, mais se poursuit d'une façon continue, serait dû à ce que les liaisons moléculaires ne se relâchent pas toutes simultanément, mais seulement petit à petit.

Après ces succès remarquables, il n’est pas étonnant que Bolzmann ait fait du principe de l'équipartition de l'énergie le centre de la théorie cinétique de la chaleur, et que, aujourd'hui encore, de nombreux physiciens inclinent vers la même manière de voir, et s'en remettent sans crainte à l'avenir, pour la solution des difficultés particulières et des contradictions qui subsistent encore. Il me sera facile cependant de montrer que ce point de vue ne peut plus se soutenir aujourd'hui, et que de nouvelles expériences, jointes à d'autres plus anciennes et connues depuis longtemps déjà, nous contraignent de retirer à la loi de l'équipartition de l'énergie son rôle de principe fondamental de l'équilibre calorifique statistique.

Je commence par les difficultés les plus anciennes. Une molécule biatomique, comme celle de l'oxygène, de l'hydrogène ou de l'azote, possède, si on considère les atomes comme des points matériels parfaitement libres, 9 degrés de liberté, savoir : les 6 composantes des vitesses des deux atomes et les 3 projections de la distance des atomes sur les trois axes de coordonnées. Or la chaleur moléculaire à volume constant n'est pas 9 , comme on devrait s'y attendre, mais seulement $ّ$. Et il en est ainsi dans tous les cas analogues. La cha- 
leur moléculaire est toujours plus petite que celle qu'on déduirait du nombre des degrés de liberté. Ce n'est d'ailleurs pas encore tout. La molécule d'un gaz monoatomique, de la vapeur de mercure par exemple, n'est certainement pas un simple point matériel. Il suffit, pour s'en convaincre, de jeter un coup d'oil sur le spectre du mercure avec ses raies fines innombrables. Si chacune de ces raies correspondait seulement à un degré de liberté, dans le sens de la théorie statistique de la chaleur, la chaleur atomique à volume constant de la vapeur de mercure ne devrait pas ètre égale à 3 , comme c'est le cas, mais dépasser 1.000 .

On comprend que ces difficultés n'aient pas échappé à Boltzmann, et qu'il ait cherché à se rendre compte de la multitude embarrassante de ces degrés de liberté, qui refusaient d'une façon si opiniâtre à se mettre en évidence par les mesures calorifiques. Or il était impossible d'interpréter les faits, de façon à éliminer ces degrés de liberté. Il essaya donc d'expliquer l'extraordinaire faiblesse de l'influence que les mouvements des atomes à l'intérieur de la molécule exercent sur la chaleur spécifique de cette molécule, par un retard dans l'établissement de l'équilibre statistique parfait. Il supposa que, pendant le temps nécessaire à la mesure de la chaleur spécifique, les vibrations des éléments de la molécule n'arrivent pas à produire des modifications sensibles, et que l'équilibre calorifique ne s'établit que plus tard et très lentement à mesure que se poursuit le mouvement de la molécule, de sorte que le phénomène n'est plus accessible à l'observation. D'après cette manière de voir, la température d'un gaz, absolument à l'abri de tout échange de chaleur avec l'extérieur, devrait lentement se modifier d'elle-même. Or on n'a jamais observé le moindre indice d'un pareil phénomène. Au contraire, la mesure des chaleurs spécifiques au moyen des rapides vibrations sonores a donné exactement les mêmes résultats que les mesures calorimétriques directes.

Encore plus difficile est la situation dans le cas des corps solides. de ceux en particulier, qui sont bons conducteurs de la chaleur et de l'électricité : les métaux. D'après la théorie nouvelle des électrons, qui s'est trouvée vérifiée dans tant de ses conséquences diverses, on admet que les porteurs de la chaleur et de l'électricité, évoluant par conductibilité, sont des électrons appelés électrons libres, qui peuvent circuler entre les molécules du métal. Si à un pareil électron on attribuait, comme il convient à un électron qui mérite réellement 
le beau nom de " libre ", les 3 degrés de liberté correspondant aux trois composantes de sa vitesse, la chaleur moléculaire d'un métal devrait toujours être sensiblement plus grande que 6 .

Dans tous les exemples apportés jusqu'ici la contradiction reste en quelque sorte latente; et l'on pouvait toujours espérer qu'une heureuse modification du principe permettrait de lever encore une fois la difficulté. La première fois que la loi de l'équipartition de l'énergie entra ouvertement en conflit avec l'expérience, ce fut lorsqu'on voulut l'appliqiaer aux lois du rayonnement du corps noir. La chaleur rayonnante nese mesure pas seulement d'une façon plus précise que la chaleur de conduction, mais encore, grâce à l'analyse spectrale, elle peut être étudiée dans ses caractères les plus délicats, alors que la chaleur de conduction se présente toujours comme un tout insécable. C'est pourquoi il était réservé aux recherches instituées dans le domaine de la chaleur rayonnante d'apporter un peu plus de clarté dans l'étude de la relation entre la température et l'énergie.

$J .-H$. Jeans a, je le crois, démontré d'une façon irréfutable que la loi de l'équipartition de l'énergie, appliquée aux phénomènes du rayonnement calorifique, conduit à une distribution de l'énergie dans le spectre normal, caractérisée par le fait que l'intensité dans le spectre est proportionnelle à la température, et inversement proportionnelle à la quatrième puissance de la longueur d'onde. Savoir : plus petite est la longueur d'onde et plus grande, par suite, est la fréquence de vibration, plus grand sera le nombre de degrés de liberté, auxquels correspond un rayonnement, distribué sur une largeur spectrale déterminće.

On voit de suite que cette loi de distribution de l'énergie ne conduit pas à une distribution de l'énergie réellement déterminée pour toute l'étendue du spectre, et qu'ellene correspond pas par conséquent à la possibilité d'aucun équilibre thermodynamique véritable. En effet, pour qu'un tel équilibre pût être réalisé,il faudraitdans tous les cas que l'intensité du spectre arrive à un maximum, puis décroisse de nouveau et aille finalement en s'évanouissant, quand décroît la longueur d'onde. Jeans n'en conclut pas néanmoins que la loi de l'équipartition de l'énergie ne pouvait pas être maintenue; mais, suivant la voie que Boltzmann avait déjà indiquée, il chercha une issue dans la considération de l'évolution dans le temps du phénomène du rayonnement. D'après lui, lorsqu'un rayonnement a lieu 
dans une enceinte vide, on n'arrive pas à un véritable équilibre thermodynamique, mais il doit se former sans cesse des radiations de très courte longueur d'onde, qui sont diffusées à l'extérieur, à la manière des rayons Röntgen durs. Mais cette conception se justifie encore plus difficilement que celle de Boltzmann. En effet, comme l'ont démontré $O$. Lummer et $E$. Pringsheim, elle est en contradiction directe a vec toutes les expériences. Et, comme toute autre issue est fermée, la généralité de la loi de l'équipartion de l'énergie se trouve battue en brèche pour la première fois.

On peut d'ailleurs le faire ressortir encore plus clairement. Les chaleurs spécifiques des corps solides présentent une tendance persistante à décroître en même temps que la température; et, tout récemment, $W$. Nernst a montré, soit expérimentalement par des mesures poursuivies jusqu'à la température d'ébullition de l'hydrogène, soit théoriquement par application de son nouveau théorème thermodynamique : les chaleurs spécifiques de tous les corps solides et liquides convergent vers une valeur infiniment petite, quand la température décrô̂t indéfiniment. C'est ainsi que la chaleur spécifique du cuivre à la température d'ébullition de l'hydrogène n'est plus que la trentième partie de sa valeur à la température ordinaire. Comment expliquer ce fait au moyen du principe de l'équipartition de l'énergie? Comment expliquer en particulier que la chaleur moléculaire d'un corps devienne plus petite que 3 ? En effet, dès qu'une molécule peut se mouvoir dans un espace, les composantes de sa vitesse constituent 3 degrés de liberté de mouvement. A la vérité, on pourrait encore supposer que, aux basses températures, les molécules d'un corps solide ou liquide s'agglomèrent en partie en groupes rigides, capables seulement de se mouvoir en bloc. De cette façon, le nombre des degrés de liberté se trouverait diminué. Mais il reste toujours tous les mouvements dont dérivent les phénomènes d'émission et d'absorption de la chaleur rayonnante de toutes les longueurs d'onde; et ceux-ci sont en tous cas d'allure très déliée, puisqu'ils correspondent à des degrés de liberté, dont le nombre surpasse certainement de plus de trois fois le nombre des molécules.

Après l'exposé de tous ces faits, il ne peut plus y avoir de doute sur l'absolue nécessité de cette conclusion : la loi de l'équipartition de l'énergie ne joue pas dans la thermodynamique le rôle fondamental qu'on lui a attribué pendant un certain temps. La question de la rela- 
tion entre la température et l'énergie se pose ainsi de nouveau avec toute son acuité. En effet, si, dans l'équilibre statistique, l'énergie moyenne n'est pas uniformément répartic entre les différents degrés de liberté, l'énergie moyenne, correspondant à un degré de liberté pris en particulier, ne peut pas être prise comme mesure de la température, attendu que dans l'état d'équilibre la température doit certainement être la même partout.

Comment faire maintenant pour sortir de ce difficile dilemme? Devons-nous tenir la loi de l'équipartition de l'énergie pour complètement fausse, et chercher quelque chose d'entièrement nouveau? Non, certes. Cette loi a en effet reçu dans un certain domaine, en particulier dans le cas des gaz monoatomiques et même jusqu'à un certain point dans le cas des corps solides, des vérifications éclatantes. Elle contient donc certainement une part de vérité. Mais elle ne contient pas toute la vérité. Nous sommes ainsi amenés à penser qu'elle constitue une généralisation illégitime d'un principe exact en lui-mème. Et, pour arriver à la généralisation exacte, nous devrons resuivre le chemin qui a conduit à la loi d'équipartition de l'énergie, retrouver le point où l'on s'est engagé dans la mauvaise voie, nous garder de nous y fourvoyer et prendre la bonne direction. Afin de mettre ce plan à exécution, nous remarquerons tout d'abord que la loi de l'équipartition de l'énergie a été tirée de l'application à l'équilibre statistique du calcul des probabilités. Nous conserverons nous aussi ce point de départ, car, sans l'introduction des considérations statistiques, il est impossible de saisir les particularités caractéristiques de l'équilibre calorifique, contrairement à ce qui a eu lieu pour l'équilibre mécanique ou électrique.

L'état d'équilibre statistique se distingue de tous les autres états possibles, correspondant à une même somme d'énergie, en ce qu'il est conditionné par un maximum de probabilité. Si deux corps, isolés pour tout le reste, peuvent échanger mutuellement leurs énergies calorifiques par conductibilité ou par rayonnement, ils seront l'un par rapport à l'autre en équilibre statistique, quand le passage de la chaleur d'un corps à l'autre ne correspondra plus à un accroissement de probabilité. Si $\mathscr{Q}_{1}=f\left(\mathscr{E}_{1}\right)$ représente la probabilité, pour que le premier corps possède l'énergie $\mathscr{E}_{1}, \mathscr{Q}_{2}=\mathscr{\psi}\left(\mathscr{E}_{2}\right)$ la probabilité pour que le deuxième corps possède l'énergie $\varepsilon_{2}$, la probabilité pour que le premier corps possède l'énergie $\mathcal{E}_{1}$ et qu'en même temps le deuxième corps possède l'énergie $\mathcal{E}_{2}$, sera repré- 
sentée par $\mathscr{Q}_{1} \mathbb{Q}_{2}$. La condition pour que cette grandeur passe par un maximum donne:

$$
d\left(\mathbb{Q}_{1} q_{2}\right)=0 \quad \text { ou } \quad \frac{d \mathscr{Q}_{1}}{\mathscr{Q}_{1}}+\frac{d \mathscr{Q}_{2}}{\mathscr{Q}_{2}}=0
$$

En ajoutant la condition permanente :

$$
d \mathfrak{\varepsilon}_{1}+d \mathfrak{\varepsilon}_{2}=0,
$$

qui exprime que l'énergie totale ne change pas, on obtient l'équation :

$$
\frac{1}{\mathscr{Q}_{1}} \frac{d \mathscr{Q}_{1}}{d \mathcal{E}_{1}}=\frac{1}{\mathscr{Q}_{2}} \frac{d \mathscr{Q}_{2}}{d \mathcal{E}_{2}}
$$

comme condition de l'équilibre statistique.

Si maintenant nous identifions l'équilibre statistique avec l'équilibre calorifique, et si nous remarquons que la condition de l'équilibre calorifique s'exprime par l'égalité de la température des deux corps, nous voyons immédiatement que l'on obtiendra un accord parfait entre la théorie statistique et la thermodynamique, en prenant d'une façon générale la grandeur :

$$
\frac{1}{d} \frac{d \mathscr{E}}{d s}=\frac{d \log \mathscr{Q}}{d s},
$$

comme mesure de la température d'un corps, et en posant par suite:

$$
k \frac{d \log \mathscr{Q}}{d \mathbb{E}}=\frac{1}{\mathscr{C}}
$$

Le fait de prendre précisément l'inverse et non pas une autre fonction de la température, provient uniquement de l'adoption de l'échelle ordinaire des températures. En principe, toute autre fonction de température nous rendrait les mêmes services. La constante $k$ dépend seulement des unités arbitrairement choisies pour la température et l'énergie.

Nous pouvons considérer l'équation (1) comme la réponse la plus générale à la question que nous avons posée de la relation entre la température et l'énergie. Elle est manifestement très prochement apparentée avec l'équation connue de la thermodynamique :

$$
\frac{1}{\tilde{E}}=\frac{d \mathrm{~S}}{d \mathbb{E}}
$$


elle a cependant une tout autre signification. L'équation de la thermodynamique pure en effet sert seulement de définition à l'entropie $\mathrm{S}$ de sorte que, prise en elle-même, elle ne représente pas une loi physique. Dans l'équation (1), au contraire, nous avons une véritable relation entre les grandeurs définies indépendamment l'une de l'autre. Nous arrivons donc par ce moyen à une définition de l'entropie différente de celle de la thermodynamique pure :

$$
\mathrm{S}=k \log (\text {, }
$$

et cette définition donne de la signification physique del'entropie une idée beaucoup plus intuitive que la définition purement thermodynamique, dont le véritable sens est saisi avec beaucoup de peine par tant dejeunes étudiants. Mais, pour le but que nous poursuivons ici, nous pouvons nous en tenir à la considération seule de la probabilité $\mathscr{D}_{1}$ et laisser complètement de côté la grandeur auxiliaire $\mathrm{S}$.

Il semble bien que, grâce à la nouvelle relation obtenue, notre problème ait fait un progrès fondamental. On pourrait même le regarder comme complètement résolu, si l'on pouvait réellement se donner la probabilité $\mathscr{Q}$ comme une fonction de l'énergie $\mathcal{E}$. Mais ceci n'est pas encore possible d'une façon générale. Il y a cependant un certain nombre de cas importants, dans lesquels on peut calculer jusqu'au bout la probabilité (2. Les méthodes, qui conduisent à de tels calculs ont été d'abord développées par Boltzmann et Gibbs, en admettant la légitimité de l'application des équations générales de Hamilton et du théorème qui en a été déduit par Liourille.

Je considère d'abord le cas d'un gaz parfait, constitué par $ォ$ molécules monoatomiques. On a alors :

$$
P=\varepsilon^{\frac{3 \Re 6}{2}} \cdot \mathrm{C}^{\mathrm{te}}
$$

où la constante ne dépend pas de l'énergie $\varepsilon$ et en substituant dans l'équation (1):

$$
\varepsilon==k \cdot \frac{33 \tau}{2} \cdot \tau \text {. }
$$

Si $k$ est connu, ou peut, en mesurant directement $\varepsilon$ et $\overleftarrow{E}$ calculer le nombre absolu $\nwarrow$ des molécules. L'énergie moyenne d'un atome est alors $\frac{3 k}{2} \tau$ et la chaleur atomique $\frac{3 k}{2}$. Elle est donc indépendante de la nature du gaz, et correspond tout à fait à ce que donne la loi 
de l'équipartition de l'énergie pour trois degrés de liberté puisque chaque degré de liberté possède la capacité calorifique $\frac{k}{2}$.

Si l'on veut calculer la probabilité $\mathscr{Q}$ pour un gaz polyatomique, il est nécessaire de faire une hypothèse sur le nombre des degrés de liberté avec lesquels atomes, ions et électrons se meuvent dans la molécule. C'est ici manifestement, qu'on s'est engagé dans une mauvaise voie, pour aboutir à la loi de l'équipartition de l'énergie. Si, en effet, on admet que les particules constitutives de la molécule se meuvent comme des points parfaitement libres, et qu'on leur applique les équations de Hamılton, on obtient pour la probabilité $Q$ une expression de la même forme absolument que dans le cas d'un gaz monoatomique. Seulement, au lieu du nombre 3 , on a le nombre $n$ des degrés de liberté de la molécule, et l'on arrive naturellement à la loi de l'équipartition de l'énergie, c'est-à-dire à une contradiction manifeste avec l'expérience.

C'est donc ici qu'il faut faire la correction. Si naturelle et si séduisante que soit l'hypothèse, faite constamment jusqu'ici, que les équations de Hamilton s'appliquent encore en toute rigueur aux phénomènes délicats qui s'accomplissent à l'intérieur des molécules et même des atomes, nous devons conclure qu'elle constitue une extrapolation condamnée par l'expérience, et supposer au contraire que le nombre des degrés de liberté dans la molécule, servant à déterminer la probabilité $\mathscr{A}$, est plus petit, et souvent beaucoup plus petit, que le nombre des éléments de la molécule. Ceci n'est possible qu'à la condition de nous faire des phénomènes intra-moléculaires une représentation tout à fait différente de celles qu'on a utilisées jusqu'ici. Nous devons alors imaginer une nouvelle hypothèse, qui ait pour effet de limiter d'une façon très notable le nombre des différents états possibles à l'intérieur de la molécule. La justification d'une telle hypothèse, en raison même de son caractère d'absolue nouveauté, ne peut se faire qu'a posteriori, et relève exclusivement de l'expérience. Au reste toute hypothèse est permise qui n'est pas en contradiction avec les lois connues de la physique. Et, comme jusqu'à présent, nous connaissons extrêmement peu de chose des procès intra-moléculaires, il reste toujours un champ très vaste à l'imagination.

Nous aurons une hypothèse qui diminue le nombre des degrés de liberté à l'intérieur d'une molécule, en supposant que les vibrations 
rapides, qui prennent naissance à l'intérieur de la molécule et produisent les phénomènes d'émission et d'absorption de chaleur, ne peuvent pas posséder une énergie quelconque, mais que leur énergie est nécessairement un multiple entier d'une certaine quantité finie $\mathfrak{E}$, qui est déterminée par les fréquences de vibration. Cette hypothèse donne, de la probabilité pour que $\mathcal{T}$ molécules possèdent l'énergie $\mathcal{E}$, l'expression:

$$
M=\frac{\left(\frac{\varepsilon}{\partial \tau_{\varepsilon}}+1\right)^{\frac{\varepsilon}{\varepsilon}+\partial \tau}}{\left(\frac{\varepsilon}{\partial \tau \varepsilon}\right)^{\frac{\varepsilon}{\varepsilon}}},
$$

et, d'après l'équation $(\mathbf{1})$, entre la température $\mathscr{E}$ et l'énergie $\varepsilon$, on a :

$$
\mathbb{L}=\frac{\mathcal{T}_{\varepsilon}}{e^{\frac{s}{k}}-1} .
$$

Cette formule permet de calculer directement l'intensité du rayonnement monochromatique de la longueur d'onde correspondante. On a donc un moyen de la vérifier en comparant le résultat du calcul avec les lois expérimentales de la distribution de l'énergie dans le spectre normal du rayonnement calorifique. L'expérience a donné jusqu'ici une concordance parfaite, si l'on fait $\varepsilon$ proportionnel à la fréquence de vibration $v$ :

$$
\varepsilon=h v .
$$

On a alors :

$$
h=6,55 \cdot 10^{-27} \mathrm{erg}-\mathrm{sec} \quad \text { et } \quad k=1,3 \pm 6 \cdot 10^{-16} \frac{\mathrm{erg}}{\text { degré }} .
$$

On voit qu'il n'est plus question d'une égale répartition de l'énergie. En effet, pour deux molécules, ayant des fréquences de vibration $\vee$ différentes, les énergies moyennes sont tout à fait différentes pour une même température. Mais quand la quantité élémentaire d'énergie $\varepsilon$ est très petite, c'est-à-dire dans le cas de vibrations très lentes, ou quand la température $\widetilde{E}$ est très élevée, on obtient:

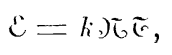

et l'on arrive à la même relation entre la température et l'énergie 
que celle qui se déduit de la loi de l'équipartition de l'énergie, en admettant deux degrés de liberté (pour l'énergie cinétique et l'énergie potentielle). En général la loi de l'équipartition de l'énergie ne s'applique pas exactement, et la température ne peut pas mesurer l'énergie moyenne d'une molécule.

A. Einstein a fait en outre l'hypothèse que, dans le cas des corps solides, l'énergie de vibration $\Sigma$ des molécules, multipliée par le facteur numérique 3 , correspondant aux trois directions de vibration possibles dans l'espace, constitue alors l'énergie calorifique totale du corps. $W$. Nernst a étudié expérimentalement, par des mesures effectućes aux basses températures, la formule caractéristique des chaleurs spécifiques qui se déduit de cette hypothèse et l'a vérifiée dans ce qu'elle a d'essentiel. L'hypothèse des éléments d'énergie a ainsi reçu une solide et toute nouvelle confirmation.

Bien qu'elle ait été jusqu'à présent vérifiée par l'expérience, cette hypothèse est encore dans sa forme actuelle susceptible de perfectionnements. En effet, la supposition que l'énergie de vibration $\varepsilon$ est un multiple entier de $\varepsilon$, entraîne la conséquence suivante. La molécule ne peut changer son énergie de vibration que par sauts discontinus. Il est alors très difficile sinon impossible de comprendre comment la molécule peut absorber d'un seul coup la quantité élémentaire d'énergie $\varepsilon$ tout entière, alors que cependant, pour l'absorption d'une quantité d'énergie finie, provenant d'un rayonnement d'intensité finie, il faut toujours une durée finie.

Il me semble donc nécessaire de modifier l'hypothèse des éléments d'énergie de la façon suivante. Seule l'émission de l'énergie se fait par à-coups, par quantités d'énergie $\varepsilon$ entières et d'après les lois du hasard; l'absorption, au contraire, se poursuit d'une manière paiffaitement continue.

On dit parfois en faveur d'une hypothèse qu'elle est utile non seulement dans les cas pour lesquels elle a été faite, mais encore dans d'autres questions. Eh bien, on peut accorder provisoirement cette bonne note à l'hypothèse des éléments d'énergie. On suppose ici, en effet, qu'une molécule ne peut émettre de l'énergie de vibration que suivant certaines quantités déterminées $s$, qu'il s'agisse de pure énergie de rayonnement comme dans le rayonnement calorifique, les rayons Röntgen et les rayons $\gamma$, ou d'un rayonnement corpusculaire, comme dans le cas des rayons cathodiques et des rayons $\alpha$ et $\beta$. Or non seulement l'hỵpothèse a été vérifiée déjà pour ce qui regarde 
DÉGOMBE. - NATURE DE LA CHALEUR NON COMPENSÉE 359

la loi du rayonnement calorifique, mais encore elle a fourni une méthode très précise pour déterminer les quantités élémentaires d'électricité et de matière, elle a donné la clef qui permet de pénétrer le sens du théorème thermodynamique de Nernst. Il semble aussi que, dans l'émission des rayons cathodiques, dans l'effet photo-électrique, de même que dans les phénomènes de la radioactivité, dont l'étrangeté confine au prodige, et auxquels les noms de Becquerel, Curie, Rutherford resteront toujours attachés, elle doive jouer un rôle fondamental.

Dirons-nous après cela que l'hypothèse des quantités élémentaires d'énergie renferme réellement toute la vérité ? Une pareille affirmation serait téméraire aussi bien que l'indice d'une vue bornée. Je crois néanmoins que cette hypothèse est plus près de la vérité que la loi de l'équipartition de l'énergie, qui, à sa lumière, apparaît seulement comme un de ses cas particuliers. C'est bien là tout ce que raisonnablement on doit exiger d'une hypothèse nouvelle. Quant au jugement définitif sur la valeur de celle-ci, c'est ici, comme dans toute question de physique, à l'expérience de le porter. 